\title{
A 12-year observation of water-soluble ions in TSP aerosols collected at a remote marine location in the western North Pacific: an outflow region of Asian dust
}

\author{
S. K. R. Boreddy and K. Kawamura
}

Correspondence to: K. Kawamura (kawamura@lowtem.hokudai.ac.jp)

The copyright of individual parts of the supplement might differ from the CC-BY 3.0 licence. 
(a)

(b)

(d)

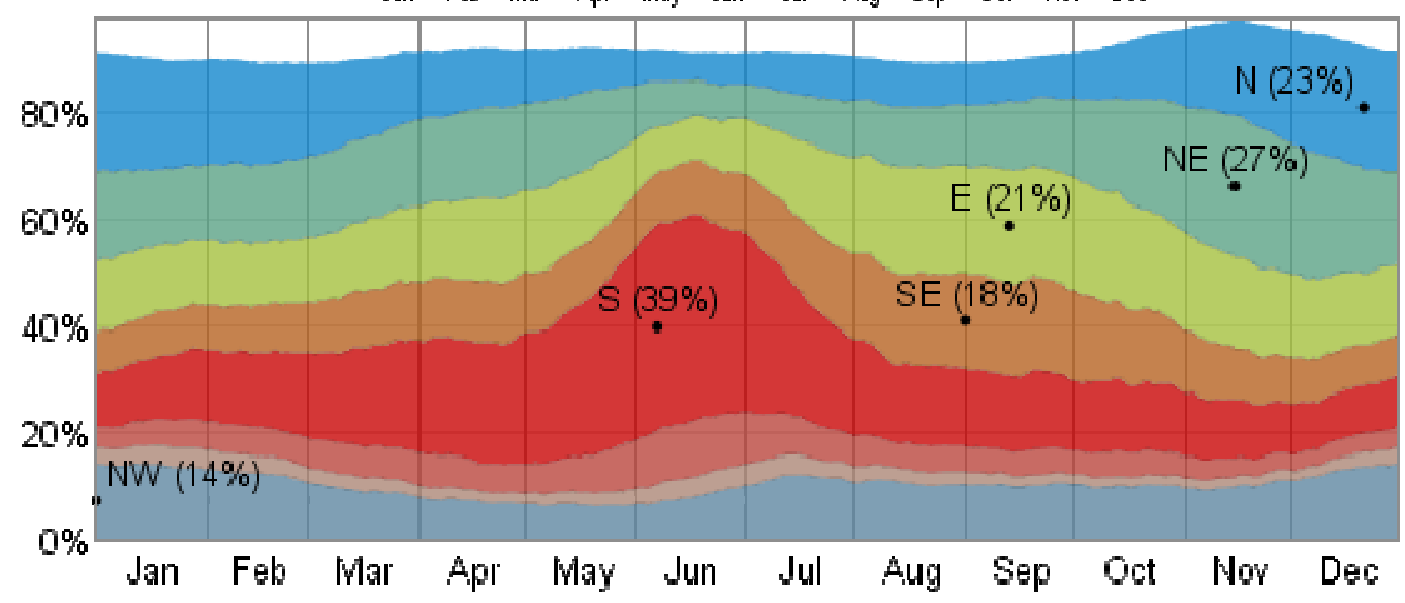

Figure S1: (a) The daily average low (blue) and high (red) temperature with percentile bands (inner band from 25th to 75th percentile, outer band from 10th to 90th percentile) (b) The average daily high (blue) and low (brown) relative humidity with percentile bands (inner bands from 25th to 75th percentile, outer bands from 10th to 90th percentile) (c) The average daily minimum (red), maximum (green), and average (black) wind speed with percentile bands (inner band from 25th to 75th percentile, outer band from 10th to 90th percentile) (d) The fraction of time spent with the wind blowing in the various directions on a daily basis. Stacked values do not always sum to $100 \%$ because the wind direction is undefined when the wind speed is zero.

This report describes the typical weather at the Chichijima (Chichijima, Japan) weather station over the course of an average year. It is based on the historical records from 1974 to 2011( http://weatherspark.com). 